\title{
Electron Beam Effects on Liquid Specimens in (S)TEM
}

Nan Jiang

Department of Physics, Arizona State University, Tempe AZ 85287-1504

Electron beam effects in (S)TEM include knock-on damage, radiolytic process and damage by induced electric field (DIEM). Among these, the DIEM mechanism dominates the beam-induced phenomena in materials which are insulators to electrons but conductors to ions [1]. The electric field is produced by net charges due to the emission of electrons, which cannot be neutralized immediately due to the low electric conductivity of the system. The strength of the induced electric field depends on dielectric properties of illuminated materials and on beam current density.

For weak electric fields, the Gibbs free energy of nucleation can be enhanced or reduced by the electric field. As we knew, for a uniform external electric field E, the modification of the Gibbs free energy by the electric field can be simplified as [2],

$$
\Delta G(N)=-k_{B} \mathrm{TN}\left(\mathrm{s}_{0}+s_{E}\right)
$$

in which $\mathrm{k}_{\mathrm{B}}$ is Boltzmann's constant, $\mathrm{T}$ is the absolute temperature, $\mathrm{N}$ is atom numbers in precipitate. Here $s_{0}$ represents the supersaturation in the absence of the electric field, and $\mathrm{s}_{\mathrm{E}}$ represents the supersaturation change due to the electric field. Fig. 1 shows the dependence of $\mathrm{SE}_{\mathrm{E}}$ on the ratio of permittivity, $\varepsilon_{\mathrm{c}} / \varepsilon_{\mathrm{m}}$, in which $\varepsilon_{\mathrm{c}}$ and $\varepsilon_{\mathrm{m}}$ are the permittivity of a nucleus and the solution, respectively. It is seen that the Gibbs free energy of nucleation may be reduced if $\varepsilon_{\mathrm{c}}>\varepsilon_{\mathrm{m}}$, or increased if $\varepsilon_{\mathrm{c}}<\varepsilon_{\mathrm{m}}$ by the electric field.

For strong electric fields, which are strong enough to overcome threshold energies for atom migration, the random Brownian diffusion is violated, resulting in ion drifting. For example, the activation energy for $\mathrm{Na}^{+}$ion in glassy materials is about $1 \mathrm{eV}$, and thus the induced electric fields can drift $\mathrm{Na}^{+}$if $\mathrm{E}>1.0 \mathrm{~V} / \mathrm{nm}$ [3]. In solution, $\mathrm{Na}^{+}$can be drifted at even weaker fields. According to MD simulations, the drifting velocities of $\mathrm{Na}^{+}$ion in water are in order of $\sim 1.0 \mathrm{~m} / \mathrm{s}$ for the electric field stronger than $\sim 0.01 \mathrm{~V} / \mathrm{nm}$ [4]. As illustrated in Fig. 2, the induced electric fields are not uniform in (S)TEM. The drifted cations and anions may separate and aggregate in different regions, resulting in the change of the local composition and thus the supersaturation. As a result, the observed precipitations in (S)TEM should be dependent on illumination conditions. In a focused-beam STEM illumination (Fig. 2), positive charges mainly concentrate within the probed region, and therefore the fields are cylindrically symmetric around the focused electron beam, and the strength drops quickly away from the beam [3]. Therefore, the metal nanoparticles may easily precipitate scattered around the probed region, but the gas bubbles form inside the probed region. In a broad-beam TEM illumination, however, the positive charges accumulate in the surface regions. The electric fields are more complicated, but in general the fields are stronger near the surfaces and the edge of the beam [5]. As a result, the metal nanoparticles can be more easily observed near the region of beam edge, while gas bubbles can be found near the window membranes of liquid cell. 
In conclusion, the observed crystal growth process in a liquid cell specimen may not be the same process of nucleation and growth seen without an electron beam. Therefore, observations from in situ liquid (S)TEM studies should not be used to support or oppose conventional nucleation and crystal growth theories. Instead, the results should be only interpreted within their own domain of relevance.

References:

[1] N. Jiang, Report on Progress in Physics 79 (2016) 016501.

[2] J. O. Isard, Phil. Mag. 35 (1977) 817-819.

[3] N. Jiang, J. Phys D: Appl. Phys. 46 (2013) 305502.

[4] S. Murad. J. Chem. Phys. 134, (2011) 114504.

[5] N. Jiang. Micron 83 (2016) 79-92.

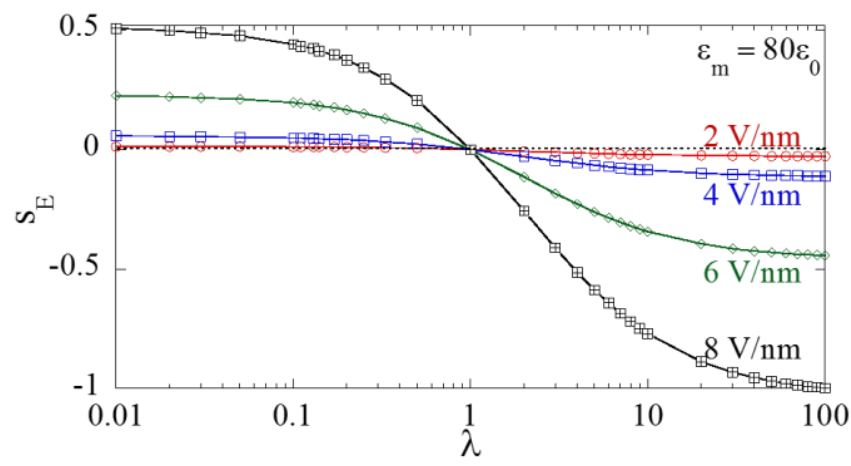

Figure 1 The calculated supersaturation, $\mathrm{s}_{\mathrm{E}}$, due to the electric field using Eq. (3), assuming that the permittivity of solution $\varepsilon_{\mathrm{m}}=80 \varepsilon_{0}$ and the radius of atom is $100 \mathrm{pm}$. $\lambda=\varepsilon_{\mathrm{c}} / \varepsilon_{\mathrm{m}}$, the ratio of permittivity of precipitation and solution.
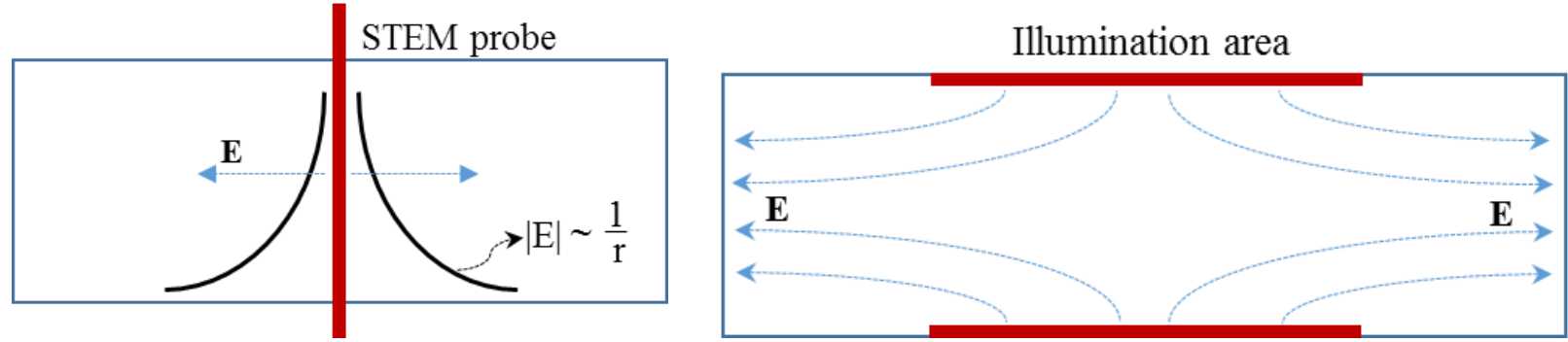

Figure 2 The drawings showing the induced electric fields under (a) STEM and (b) TEM illumination. Red bars represent the charged regions, and arrows indicate the field directions. 